\title{
Tidal flow variability measured by impedance pneumography relates to childhood asthma risk
}

\author{
Ville-Pekka Seppä ${ }^{1}$, Anna S. Pelkonen², Anne Kotaniemi-Syrjänen², Jari Viik , \\ Mika J. Mäkelä and L. Pekka Malmberg ${ }^{2}$
}

Affiliations: ${ }^{1}$ Dept of Electronics and Communications Engineering, Tampere University of Technology, BioMediTech, Tampere, Finland. ${ }^{2}$ Dept of Allergology, University of Helsinki and Helsinki University Hospital, Helsinki, Finland.

Correspondence: Ville-Pekka Seppä, Dept of Electronics and Communications Engineering, Tampere University of Technology, BioMediTech, Biokatu 6, 33560 Tampere, Finland. E-mail: vpsवiki.fi

ABSTRACT Lung function variability is a fundamental feature of asthma but has been difficult to quantify in children due to methodological limitations. We assessed the feasibility and clinical implications of overnight flow variability measurement at home using impedance pneumography in young children.

44 children aged 3-7 years with recurrent or persistent lower airway symptoms were recruited. Patients were divided into high- or lower-risk groups (HR and LR groups) based on their risk of asthma (modified Asthma Predictive Index), and a third group was formed of children who had a history of wheeze and who were treated with inhaled corticosteroids (ICS group). Tidal volume and the derived flow were recorded through skin electrodes using impedance pneumography at home during sleep. Quantities describing overnight change in expiratory flow-volume minimum curve shape correlation (CSRmin) and respiratory chaoticity (minimum noise limit (NLmin)) were derived.

Recordings were successful in 34 children. CSRmin differed between the HR and LR groups $(p=0.002)$ and between the HR and ICS groups $(p=0.003)$, indicating a stronger change in flow profile shape in the HR group. NLmin differed between the HR and LR groups $(p=0.014)$, indicating momentarily lowered chaoticity in the HR group.

Impedance pneumography was found feasible for quantifying nocturnal lung function variability and the measured variability was associated with risk of asthma in young children.

@ERSpublications

Impedance pneumography enables home monitoring of lung function variability relating to risk of asthma in children http://ow.ly/XFWtD

This article has been revised according to the correction published in the July 2016 issue of the European Respiratory Journal. This article has supplementary material available from erj.ersjournals.com

Received: June 232015 | Accepted after revision: Jan 242016 | First published online: March 172016

Support statement: This study received grants from Tampere Tuberculosis Foundation, Helsinki University Research Funds, the Finnish Medical Foundation, Foundation for Paediatric Research, Foundation for Allergy Research, Finnish Society for Allergy and Immunology, Instrumentarium Science Foundation, Nummela Sanatorium Foundation, Finnish Funding Agency for Innovation and the Sigrid Juselius Foundation. Funding information for this article has been deposited with FundRef.

Conflict of interest: Disclosures can be found alongside the online version of this article at erj.ersjournals.com

Copyright @ERS 2016 


\section{Introduction}

Temporal variability of lung function is one of the fundamental characteristics of asthma. This variation, which is a result of complex and nonlinear behaviour of the respiratory system fluctuating with time, driven by multiple interacting systems, relates to acute conditions but may also predict future exacerbations and disease progression [1]. The variability is witnessed not only as characteristic episodic or diurnal airway narrowing, but also as other phenomena, e.g. homeokinetic fluctuations of airway calibre, volume-dependent variations during the breathing cycle as well as changes in the breathing pattern driven by respiratory control. Lung function variation in adult patients with obstructive lung diseases has been successfully assessed with various measures and at different time scales ranging from a few minutes recording of respiratory flow [2-5] (decreased variation) or mechanical impedance [6-8] (increased variation) to months-long peak expiratory flow monitoring [9]. The variation has been assessed through descriptive statistics, but also through more complex dynamic measures that describe fractality and chaos. Common techniques require direct airway access and/or respiratory manoeuvres and are thus not best suited for young children, but indirect techniques that sensor chest wall movements, such as respiratory inductive plethysmography, enable the assessment of complex breathing patterns and respiratory control even in sleeping small infants [10-13]. However, since they have only been capable of assessing the rhythmic/timing properties of breathing patterns instead of accurately characterising the flow-volume curve shape, the possibilities to assess diurnal lung function variability in asthma have been limited in young children. It is therefore unclear how the fluctuating pattern of the respiratory system relates to symptoms and their persistence at this age.

Impedance pneumography is a technique for measuring instantaneous lung aeration changes (breathing) as changes in the thoracic electrical impedance through skin electrodes. The underlying phenomenon has been known for almost a decade, but its main clinical use has been to monitor respiratory rate in intensive care settings. However, recent technical advancements in impedance pneumography signal processing [14] and electrode placement [15] have enabled impedance pneumography to be used for accurate noninvasive respiratory flow signal measurement. It is the only ambulatory, noninvasive method that has shown high flow signal agreement with direct pneumotachography, enabling tidal flow-volume curve assessment in young children even during induced airway obstruction [16].

The main objectives of this study were to assess this new technical approach in terms of its practical feasibility and the ability to characterise flow variability in clinical material. Overnight home impedance pneumography recordings in young children with lower respiratory symptoms were investigated under stable clinical conditions in an attempt to monitor time-varying changes in lung function, especially night-time worsening hypothetically reflected as slow overnight change in tidal flow-volume curve shape and as reduced chaotic fluctuation of flow during possible episodes of obstruction. We hypothesised that these measures of variability are related to respiratory symptoms and the risk of persistent asthma.

\section{Material and methods}

See the online supplementary material for additional details about the methods used.

\section{Study subjects}

We recruited 44 children aged 3-7 years who were referred to the Paediatric Allergy Unit of Helsinki University Hospital because of recurrent or persistent lower respiratory tract symptoms. Detailed subject characteristics are summarised in table 1. All study subjects were born full-term and had a history of recurrent or persistent respiratory symptoms (wheeze, cough and/or shortness of breath).

The probability of persistent asthma in each of the study subjects was estimated by using the modified Asthma Predictive Index (mAPI) [17]. Children with a history of multiple episodes of wheeze and who fulfilled one of the major criteria (parental history of asthma, atopic dermatitis, sensitisation to respiratory allergens) or two minor criteria (sensitisation to milk, egg or peanuts, wheezing unrelated to colds, blood eosinophilia) were considered to have a high risk of asthma (HR group). The group with lower asthma risk (LR group) consisted of subgroups of children with a history of wheeze but who did not fulfil the additional risk factor criteria of mAPI, children who did not have a confirmed history of wheeze but had risk factors of the additional criteria of mAPI and children who had neither a history of wheeze nor other additional risk factors. Another subgroup of children had a history of wheeze but was on inhaled corticosteroids at the time of study. Since regular maintenance medication may modify disease activity and thereby lung function variability, this group was analysed separately (ICS group). The children in the HR and LR groups had no regular asthma medication, and none of the children were given bronchodilators during home recordings.

The study was approved by an institutional paediatric ethics review board and informed written consent was received from guardians of all patients. 
TABLE 1 Characteristics of study children for whom impedance pneumography recordings were technically successful

\begin{tabular}{lccccr} 
& HR & LR & ICS & All & p-value \\
\hline Subjects & 13 & 14 & 7 & 34 & \\
Male & $5(39)$ & $6(43)$ & $4(57)$ & $15(44)$ & 0.72 \\
Age years & $4.6(3.4-6.8)$ & $5.0(3.4-6.6)$ & $5.1(3.8-6.7)$ & $4.9(3.4-6.8)$ & 0.82 \\
Height cm & $110(94-126)$ & $111(96-121)$ & $108(104-127)$ & $109(94-127)$ & 0.98 \\
Gestational age weeks & $40(38-41)$ & $41(38-42)$ & $40(39-42)$ & $40(38-42)$ & 0.43 \\
Birthweight kg & $3.6(2.8-4.6)$ & $3.6(2.1-5.2)$ & $3.5(3.0-4.1)$ & $3.5(2.1-5.2)$ & 0.66 \\
Wheez"\#, & $13(100)$ & $5(36)$ & $7(100)$ & $26(77)$ & $<0.01$ \\
Allergic rhinitis & $3(23)$ & $2(14)$ & $2(29)$ & $7(21)$ & 0.72 \\
Atopic dermatitis & $7(54)$ & $3(21)$ & $7(100)$ & $17(50)$ & $<0.01$ \\
SPT positivity & $9(69)$ & $3(21)$ & $5(71)$ & $17(50)$ & 0.02 \\
Parental asthma & $6(46)$ & $4(28)$ & $4(57)$ & $14(41)$ & 0.41 \\
Parental smoking & $1(8)$ & $6(43)$ & $2(29)$ & $9(26)$ & 0.12 \\
Rrs5 z-score & $-0.1(-1.4-1.8)$ & $0.0(-1.8-3.6)$ & $0.3(-3.6-0.9)$ & $-0.1(-3.6-3.6)$ & 0.80 \\
Xrs5 z-score & $0.2(-2.4-1.6)$ & $0.2(-6.1-1.9)$ & $-0.5(-1.6-3.7)$ & $0.2(-6.1-3.7)$ & 0.22 \\
$\Delta R$ Rrs5ex \%" & $35(17-133)$ & $8(-7-61)$ & & $24(-7-133)$ & 0.04 \\
\hline
\end{tabular}

Data are presented as $\mathrm{n}, \mathrm{n}(\%)$ or median (range), unless otherwise stated. HR: high-risk group; LR: lower-risk group; ICS: inhaled corticosteroid group; SPT: skin-prick test; Rrs5: respiratory resistance at $5 \mathrm{~Hz} ; X_{\mathrm{rs} 5}$ : respiratory reactance at $5 \mathrm{~Hz} ; \Delta R \mathrm{rs5ex}$ : exercise-induced change of $\operatorname{Rrs} 5$ ( $\mathrm{n}=15$ for all). $\mathrm{p}$-values between groups determined by Kruskal-Wallis test (continuous variables) or Chi-squared/Fisher's exact test (categorical variables). Symbols denote post hoc differences between paired groups with $p<0.05$ after Bonferroni correction: "\# : HR versus LR; ๆ: LR versus ICS.

\section{Study design}

The children were recruited consecutively after clinical assessment by a paediatric allergist, lung function measurements, and skin-prick testing. For eligible children with an informed written parental consent, an overnight recording with impedance pneumography at home was arranged. All the recordings were made under a stable clinical state, without any wheezing exacerbations within 2 weeks.

\section{Measurement methods \\ Oscillometric lung function measurement}

All the study subjects underwent lung function testing by using the oscillometric technique (MasterScreen; CareFusion, Würzburg, Germany). The methodology has been previously described in detail [18]. Baseline respiratory resistance and reactance at $5 \mathrm{~Hz}\left(R_{\mathrm{rs} 5}\right.$ and $\left.\mathrm{Xrs}_{\mathrm{rs}}\right)$ in the study groups were expressed as height adjusted $\mathrm{z}$-scores [18]. A standardised running test was performed in a subgroup of children $(\mathrm{n}=15)$, as described previously [19]. The severity of exercise-induced bronchoconstriction was expressed as the percentage increase of respiratory resistance after running $(\Delta R \mathrm{rs} 5 \mathrm{ex})$.

\section{Impedance pneumography measurement}

The overnight signals were acquired using custom-designed recording devices (design of Tampere University of Technology, Finland). The four skin electrodes were placed bilaterally on the thorax and arms close to the armpit yielding high linearity between impedance and lung volume (figure 1) [15]. Parents kept a diary of sleeping time, eventual symptoms and medication at home.

No calibration was needed for impedance pneumography in this use, but the linearity between differentiated impedance pneumography signal and mouth flow signal was ensured in sitting and laying positions using a pneumotachograph (Pro; Medikro, Kuopio, Finland) at the clinic.

\section{Impedance pneumography signal pre-processing}

All signal processing and statistical analyses were performed using MATLAB software (MATLAB R2015a; MathWorks, Natick, MA, USA). One experienced investigator who was blind to patient information screened the signals manually for sections of motion or other distortion (such as induced by cough, movement or talking) that were left out of further processing and analysis. The selected sections were then filtered with a specifically developed algorithm [14] to remove the cardiogenic impedance changes. The naturally lung volume-oriented impedance pneumography signal was differentiated to yield a flow-oriented impedance pneumography signal. 


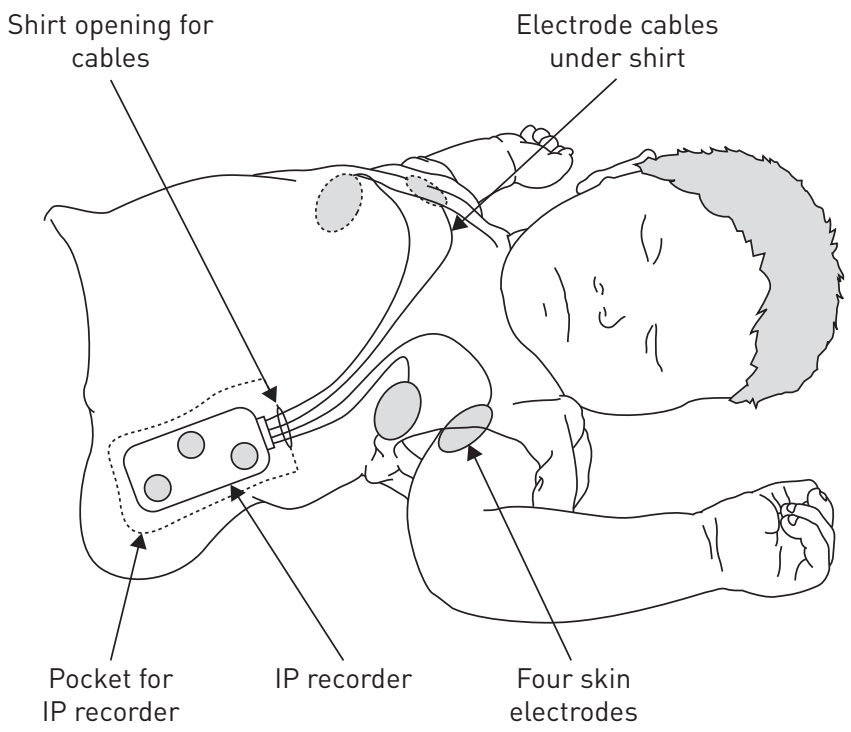

FIGURE 1 Schematic presentation of impedance pneumography (IP) recording.

\section{Measurement analysis}

The main outcome measures of the study were two impedance pneumography-derived parameters, i.e. curve shape correlation (CSR) and noise limit (NL), which were analysed with respect to the three patient groups. The most referenced tidal breathing variable, the ratio of time to peak tidal expiratory flow/total expiratory time $(t \mathrm{PTEF} / \mathrm{tE})[20]$, was also derived.

\section{Impedance pneumography signal analysis approach 1 (CSR)}

The impedance pneumography-derived volume and flow signals were cut into individual breaths and were averaged in the flow-volume domain using a window of 20 breaths that was moved in overlapping steps of 5 breaths. After averaging, the curves were normalised in flow and volume to range $0-1$.

These pre-averaged flow-volume curves were then further averaged in a longer, 2-h window by taking mean. A window length of $2 \mathrm{~h}$ was considered to extend over sleep stage intervals in children and thus minimise the possible effect of sleep stages. The following procedure for finding CSR values is illustrated in figures 2 and 3. One window was moved in steps of $10 \mathrm{~min}$ between 00:00 and 04:00 h (early set), and another window between 02:00 am and 06:00 h (late set), both time sections thus yielding 13 averaged flow-volume curves. Pearson's linear correlation coefficient was determined between all $13 \times 13$ averaged curves. Since the later part of the expiration is less affected by muscle control and thus better reflects the mechanical properties (time constant) of the respiratory system [21, 22], the correlation was calculated using only the $50-100 \%$ of expired volume part of the curve (figure 3 ). The smallest encountered curve correlation, denoting the highest overnight change in the curve shape, is reported as CSRmin for each patient. See figure 3 for examples.

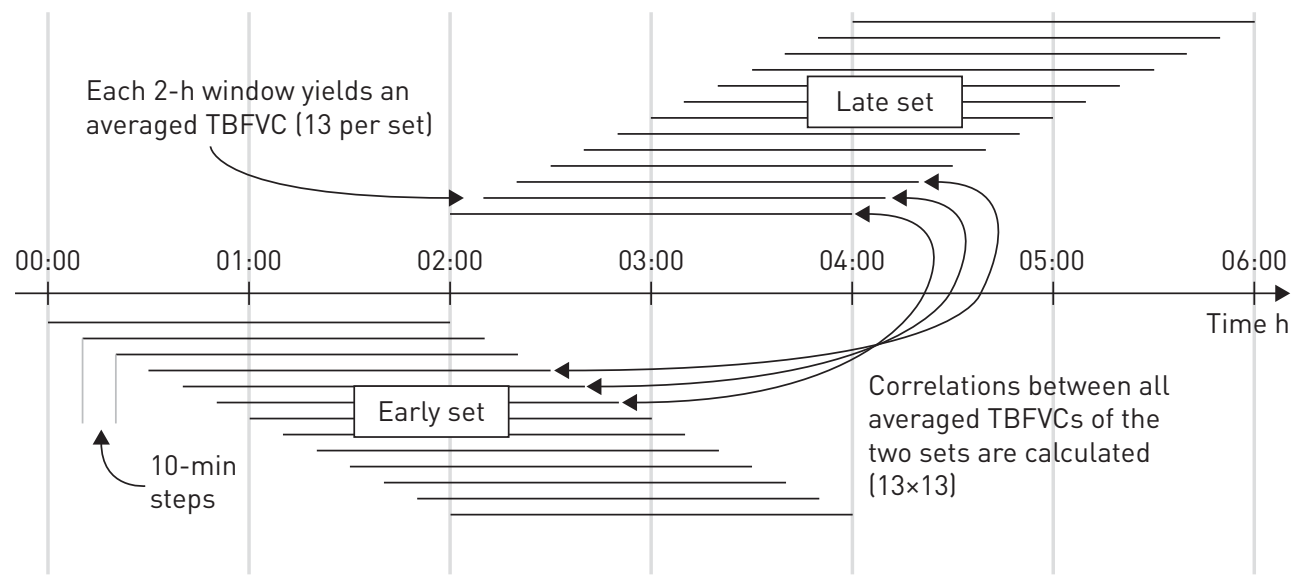

FIGURE 2 Illustration of the method for finding the minimum curve shape correlation between two averaged tidal breathing flow-volume curves (TBFVC). 


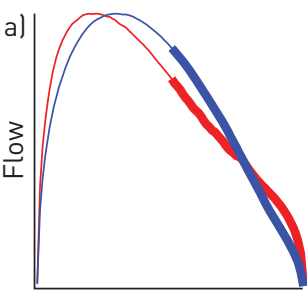

Volume CSRmin $=0.9913$

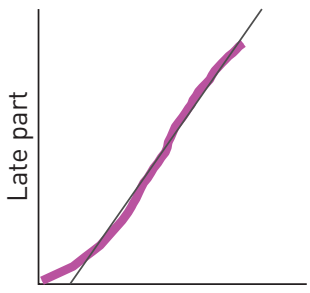

Early part

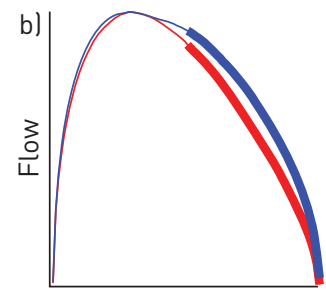

Volume $\mathrm{CSR} \min =0.9952$

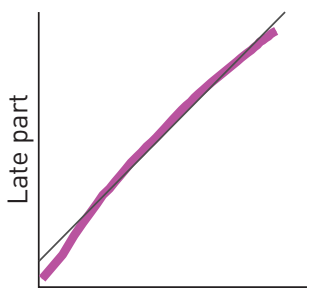

Early part

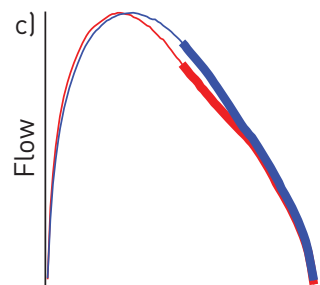

Volume CSRmin $=0.9983$

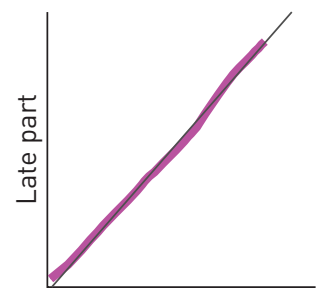

Early part

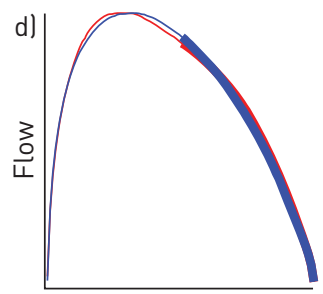

Volume $C S R_{\min }=0.9991$

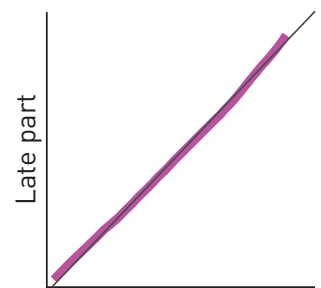

Early part

FIGURE 3 Illustration of the minimum curve shape correlation (CSRmin) with four examples of different CSRmin values from $a, b)$ two patients from the high-risk group and $c$, d) two patients from the lower-risk group. Upper curves are averaged (2-h moving window in 10-min steps) and normalised expiratory tidal breathing flow-volume curves from the first 100:00-04:00 h, blue) and later 102:00-06:00 h, red) part of the night selected to have the lowest mutual correlation. CSRmin is given as the Pearson linear correlation of the later parts $(50-100 \%$ of expired volume, thick line) of expiratory flow-volume curves as illustrated in the lower curves. Straight line denotes the best line fit $(r=1.0000)$.

Impedance pneumography signal analysis approach 2 (NL)

The impedance pneumography-derived flow signal chaoticity was defined by calculating NL using the noise titration method, which has been described in detail by Poon and BARAHONA [23]. Simplified, the algorithm tests how much white noise can be added to the signal before chaoticity can no longer be detected. A higher value denotes stronger chaos. NL was calculated for each manually defined continuous nondistorted data section lasting at least $5 \mathrm{~min}$. For each patient, the mean NL of all sections is reported as NLmean and the minimum value as NLmin. See figure 4 for examples.

\section{Statistical methods}

Differences between groups were assessed using the Chi-squared test for categorical variables and KruskalWallis or Wilcoxon rank-sum test for continuous variables. For the Chi-squared and Kruskal-Wallis tests, a post hoc analysis was performed using Fisher's exact test and Dunn's test, respectively. Correlations between continuous variables were calculated using Spearman's rank correlation. Probabilities in multiple comparisons were adjusted using the Bonferroni method where appropriate.

\section{Results}

Successful impedance pneumography recordings were obtained in 34 children. The reasons for failed recordings were electrodes coming loose $(n=5)$, patient taking off electrodes or turning off the device $(n=4)$, forgetting to turn on the device $(n=1)$ and electrodes removed by parents due to itching $(n=1)$.

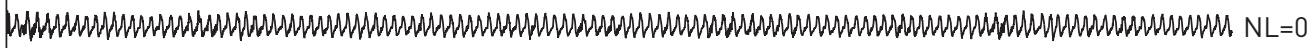

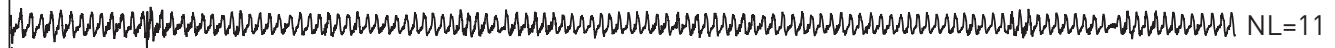

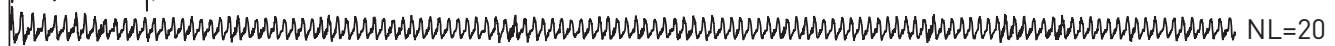

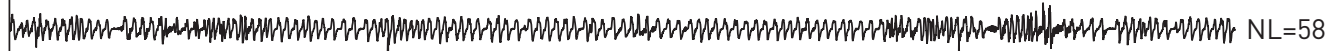

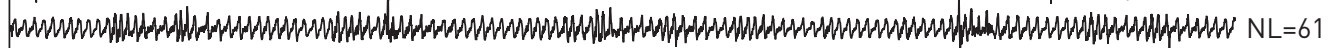

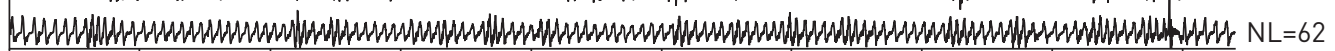

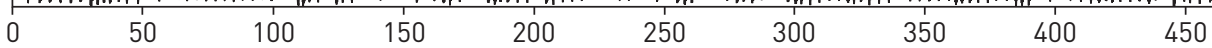

$$
\begin{aligned}
& \text { Time s }
\end{aligned}
$$

FIGURE 4 Illustration of the noise limit (NL) with excerpts from six impedance pneumography-derived flow signal segments having varying levels of NL. All signals are from one patient of the high-risk group at different times during the night. The top three segments with low NL values are seemingly less variable and more mechanistic. 
Thus, the final sample of successful recordings included 13 subjects in the HR group, 14 subjects in the LR group and seven subjects in the ICS group (table 1). The lung function results in the study groups are included in table 1 .

Children in the HR group showed significantly lower CSRmin and NLmin as compared with those in the LR group, and lower CSRmin compared with those in the ICS group (table 2 and figure 5). The association between CSRmin was found to be significant for NLmean, but not for NLmin after Bonferroni correction for multiple comparisons (table 3 and figure 6). Baseline lung function assessed with the oscillometric technique was not related to either NL or CSRmin. However, in those patients $(n=15)$ who underwent exercise challenge test, a trend was observed in CSRmin and NLmin with the severity of exercise-induced bronchoconstriction, but after Bonferroni correction, the association was not statistically significant (table 3).

Median (range) $t \mathrm{PTEF} / \mathrm{tE}$ values of averages of 1 - $\mathrm{h}$ windows through the night were $0.21(0.16-0.25), 0.19$ $(0.18-0.32)$, and $0.20(0.16-0.26)$ for the HR, LR and ICS groups, respectively. The individual minimum, maximum and standard deviation values of $t \mathrm{PTEF} / \mathrm{tE}$ of the 1 - $\mathrm{h}$ averages were also assessed, but no significant differences between groups were observed $(\mathrm{p}>0.3$ for all). However, the mean $t \mathrm{PTEF} / \mathrm{tE}$ correlated with NLmean $(\mathrm{r}=-0.50, \mathrm{p}=0.002)$, the maximum $t \mathrm{PTEF} / \mathrm{tE}$ correlated with NLmin $(\mathrm{r}=-0.41$, $\mathrm{p}=0.015)$ and NLmean $(\mathrm{r}=-0.52, \mathrm{p}=0.002)$, and the standard deviation of $t \mathrm{PTEF} / \mathrm{tE}$ correlated with NLmin $(\mathrm{r}=-0.63, \mathrm{p}=0.000)$ and NLmean $(\mathrm{r}=-0.47, \mathrm{p}=0.005)$. In addition $t \mathrm{PTEF} / \mathrm{tE}$ was derived from the same averaged tidal breathing flow-volume curves produced for CSR analysis and largest overnight difference in $t \mathrm{PTEF} / \mathrm{tE}$ was established, but no significant between-group differences were found.

\section{Discussion}

In this pilot trial, we demonstrated the use of an application based on impedance pneumography capable of recording overnight tidal flow. Indices of flow variability were derived from the recorded signal and these were shown to associate with the clinical manifestation of disease in young children with respiratory symptoms.

10 out of 44 attempts to measure impedance pneumography overnight failed, with the most common reason being electrodes coming off $(n=5)$. This could have been reduced by choosing an electrode better suited to long-term monitoring, and rehearsing the technique of electrode and electrode wire securing using adhesive tape. The few cases where the child turned off the recording device could be avoided by better design or shielding of the device.

The respiration-induced variations in the electrical impedance of the thorax that can be measured through electrodes placed on the skin surface were discovered nearly a century ago, but the clinical use of the phenomenon has been quite modest. Most noninvasive respiration measurement methods are based on chest wall movement, whereas in impedance pneumography the signal stems at least partially from the actual lung tissue aeration affecting the conductivity of the thorax [24]. Recent discoveries related to the impedance pneumography signal processing technique [14] and skin electrode locations [15] have now enabled impedance pneumography to accurately record shapes of respiratory flow profiles, as in this study. Impedance pneumography performance has been validated against direct pneumotachography in healthy adults under respiratory loading [25] and in preschool children during methacholine-induced airway obstruction [16].

Although tidal breathing is the output of a complex neuromechanical system, the majority of tidal breathing studies have assumed that obstruction would manifest similarly in all individuals. The new approach presented here, i.e. CSR, does not rely on that assumption. It merely tells us whether there is any change in the (later part of the) tidal breathing flow-volume curve, regardless of its type or direction. Neither the mean nor the variability of the most referenced tidal breathing parameter, i.e. $t \mathrm{PTEF} / \mathrm{tE}$, distinguished between groups.

\begin{tabular}{|c|c|c|c|c|}
\hline & HR & LR & ICS & $p$-value \\
\hline Subjects & 13 & 14 & 7 & \\
\hline $\mathrm{CSR}_{\min } \#$, & $0.995(0.984-0.999)$ & $0.998(0.994-0.999)^{+}$ & 0.998 (0.997-0.999) & 0.002 \\
\hline NLmin ${ }^{\#}$ & $14.3(0.00-48.7)$ & $30.3(0.00-42.7)$ & $26.7(0.00-38.0)$ & 0.03 \\
\hline NLmean & $42.2(30.8-59.2)$ & $49.8(26.3-58.5)$ & $44.8(27.8-55.7)$ & 0.15 \\
\hline
\end{tabular}

Data are presented as $\mathrm{n}$ or median (range), unless otherwise stated. HR: high-risk group; LR: lower-risk group; ICS: inhaled corticosteroid group; CSRmin: minimum curve shape correlation; NLmin: minimum noise limit; NLmean: mean noise limit. Symbols denote significant difference in Dunn's post hoc test between groups: ${ }^{\#}$ : HR versus LR; ${ }^{\text {ๆ: }}$ HR versus ICS. ${ }^{+}: n=13$. 
a)

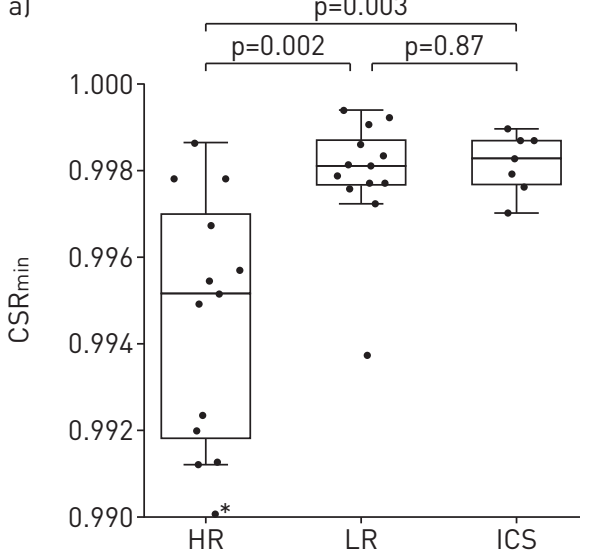

b)

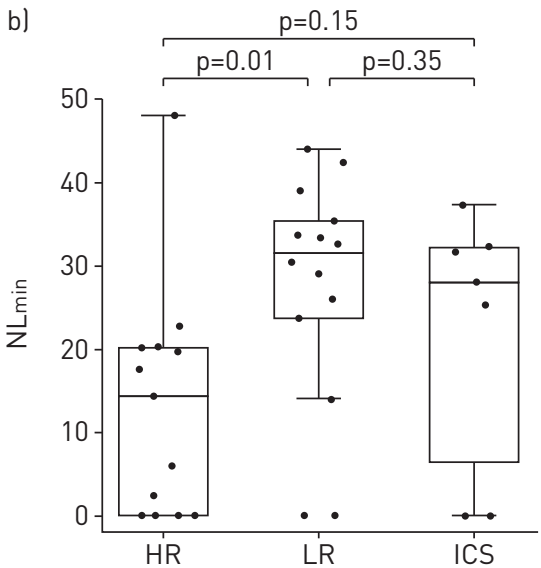

FIGURE 5 Values of a) minimum curve shape correlation (CSRmin) and b) minimum noise limit (NLmin) with median and quartiles and individual patient as dots. HR: high-risk group; LR: lower-risk group; ICS: inhaled corticosteroid group. p-values as given by Wilcoxon's rank-sum test. The asterisk denotes an individual outside of the scale (CSRmin=0.984).

It may be speculated that the novel approach of CSR that focuses only on the later, passive, part of exhalation reflects the mechanical properties of the respiratory system better than $t \mathrm{PTEF} / \mathrm{tE}$, which is influenced by the early, controlled, part of the expiration. Indeed, VAN DER ENT et al. [26] showed that $t \mathrm{PTEF} / \mathrm{tE}$ is mostly determined by activity of inspiratory muscles whose contraction extends to early expiration in healthy subjects [21].

Reduced flow signal complexity has been associated with chronic airway obstruction in adults $[2,4,5]$. Now studied for the first time in an overnight setting and noninvasively, the general overnight level of chaoticity (NLmean) did not clearly distinguish between the groups; instead, the HR group exhibited short periods of low chaoticity (NLmin), which may represent low variability during episodes of airway obstruction and highlight the benefits of a long-term ambulatory assessment. Whereas CSRmin may be an index of overall airway lability and relate to nocturnal progression of obstruction, NL is related to the nonlinear dynamics of the respiratory system in general. These measures (CSR and NL) may thereby have different long-term correlations with asthma phenotype, including predisposition for exacerbations and progression of asthma. It is difficult to estimate the contribution of neural control and airway mechanics, but interestingly both measures of interest tended to be associated with changes in airway mechanics induced by exercise, CSRmin more closely so.

A conventional method to characterise lung function variation in asthma is the recording of diurnal peak expiratory flow (PEF) rates. The relationship between PEF variation, symptoms and disease severity in childhood asthma is weak [27], and the measurements are strongly effort dependent and therefore not usually reliable in young children. Portable devices which measure interrupter resistance have been validated for preschool children, but used mainly to monitor bronchodilator responses [28, 29]. An increase of short-term variability of mechanical impedance by using the forced oscillation technique has been shown to be a feature of asthma that is more sensitive than abnormal baseline lung function [30]. Accordingly, despite significant differences in lung function variation between study groups, we found similar baseline lung function assessed by impulse oscillometry.

TABLE 3 Spearman rank correlations between impedance pneumography and oscillometric variables (z-scores)

\begin{tabular}{|c|c|c|c|c|c|}
\hline & NLmin & NLmean & Rrs5 & $X_{\text {rs5 }}$ & $\Delta R$ rs5ex \\
\hline CSRmin & 0.48 & $0.57^{*}$ & 0.03 & -0.06 & -0.55 \\
\hline NLmin & & $0.85^{* *}$ & -0.04 & -0.03 & -0.43 \\
\hline NLmean & & & -0.01 & -0.06 & -0.15 \\
\hline Rrs5 & & & & $-0.62^{* *}$ & -0.19 \\
\hline Xrs5 & & & & & -0.02 \\
\hline
\end{tabular}

NLmin: minimum noise limit; NLmean: mean noise limit; Rrs5: respiratory resistance at $5 \mathrm{~Hz}$; $X_{\mathrm{rs} 5}$ : respiratory reactance at $5 \mathrm{~Hz} ; \Delta R$ rs5ex: exercise-induced change of $R$ rs5; CSRmin: minimum curve shape correlation. *: $p<0.01 ;{ }^{* *}: \mathrm{p}<0.001$ after Bonferroni correction. 


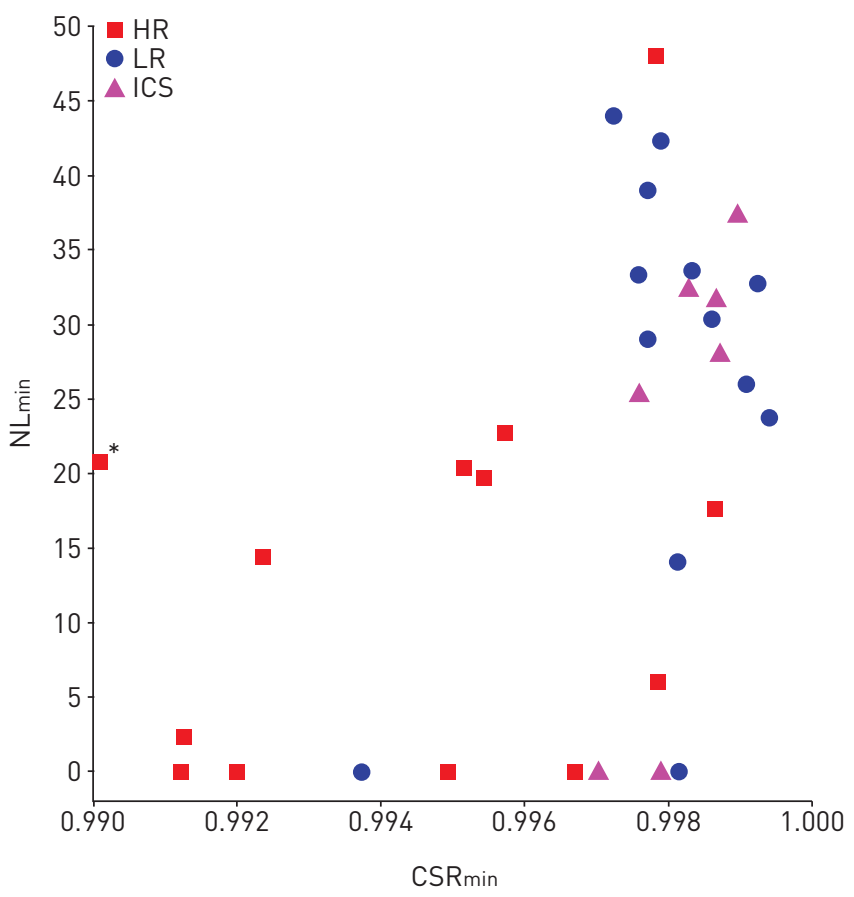

FIGURE 6 The relationship between minimum curve shape correlation (CSRmin) and minimum noise limit (NLmin) with each point representing one patient. HR: high-risk group; LR: lower-risk group; ICS: inhaled corticosteroid group. Spearman's rank correlation $r=0.479$ ( $p=0.07$ after Bonferroni correction). The asterisk denotes an individual outside of the scale (CSRmin=0.984).

The present study differs from the previous studies not only with regard to the biosignal studied, but also by characterising the spontaneous fluctuation of airflow during sleep without interference by a mask or mouthpiece to measure output of the respiratory system. Impedance pneumography also enables us to explore the complexity of asthma by continuous monitoring of lung function. FREY and SUKI [1] have pointed out that fluctuation analysis based on quantification of the long-term temporal history of lung function can be used to assess the risk of future asthma episodes and disease progression. In their analyses, they used data of diurnal PEF variation over several months [9], which may be impractical in clinical settings. Due to the fractal geometry of the nonlinear behaviour of the respiratory system [31], shorter periods as in the present study may be clinically useful when lung function is monitored continuously.

In measurement analysis of CSRmin, we used window lengths that should be long enough to extend over sleep stage intervals in children [32] in order to overcome the variation in airway resistance potentially caused by changing sleep stages [33]. Moreover, we observed that there were no differences between the groups in the amount or duration of acceptable impedance pneumography signal segments during the night. Our measurements did not include polysomnographic instrumentation such as electroencephalography and thus we cannot entirely rule out the possible contribution of sleep stages to our findings. Sleep stages differ with respect to respiratory rate, tidal volume, flow rates and their variability [34-36]. However, attributing our findings to sleep architecture differences would imply that sleep is fundamentally different between lower- and high-risk asthma patients. There is evidence of minor sleep differences between asthmatics and controls [37, 38], but to our understanding these are not large enough to support the substantial group difference we discovered.

The study sample was limited in number and reflects the heterogeneity of wheezing disorders in clinical settings. Failure to demonstrate some statistical differences, i.e. between the ICS and LR groups in terms of NL, may relate to small sample size. Due to lack of a healthy control group we were not able to define any normal ranges for the investigated parameters nor rule out whether the study subjects in the LR group would also show some abnormal lung function variability. However, management and follow-up of wheezy children should be targeted to those with the highest risk of asthma and the present results may, therefore, have clinical implications. Our results demonstrate that variability of lung function is increased already at an early age in children with a high risk of asthma and that this may be a more prominent feature than baseline lung function. The differences between the HR and ICS groups suggest that control medication modifies disease activity, and thereby lung function variability. Due to the cross-sectional design of the study, we have demonstrated here only an association between clinical asthma risk index and 
tidal flow variability. The assessment of the predictive value of the investigated parameters, as well as responses for treatment, need confirmation in a longitudinal design.

We conclude that impedance pneumography provides a novel and feasible tool to assess temporal variability of lung function in young children. This variation is related to respiratory symptoms and the risk of persistent asthma.

\section{Acknowledgements}

The authors wish to thank Mr Anssi Koivuselkä (Research Nurse, Paediatric Allergy Unit, Helsinki University Hospital, Helsinki, Finland) for practical work with patients and Dr Chi-Sang Poon (Principal Research Scientist, Massachusetts Institute of Technology, Cambridge, MA, USA) for technical assistance with the noise titration method.

\section{References}

$1 \quad$ Frey U, Suki B. Complexity of chronic asthma and chronic obstructive pulmonary disease: implications for risk assessment, and disease progression and control. Lancet 2008; 372: 1088-1099.

2 Veiga J, Lopes AJ, Jansen JM, et al. Airflow pattern complexity and airway obstruction in asthma. J Appl Physiol 2011; 111: 412-419.

3 Samara Z, Raux M, Fiamma M-N, et al. Effects of inspiratory loading on the chaotic dynamics of ventilatory flow in humans. Respir Physiol Neurobiol 2009; 165: 82-89.

4 Dames KK, Lopes AJ, de Melo PL. Airflow pattern complexity during resting breathing in patients with COPD: effect of airway obstruction. Respir Physiol Neurobiol 2014; 192: 39-47.

5 Teulier M, Fiamma M-N, Straus C, et al. Acute bronchodilation increases ventilatory complexity during resting breathing in stable COPD: toward mathematical biomarkers of ventilatory function? Respir Physiol Neurobiol 2013; 185: 477-480.

6 Gonem S, Umar I, Burke D, et al. Airway impedance entropy and exacerbations in severe asthma. Eur Respir J 2012; 40: 1156-1163.

7 Muskulus M, Slats AM, Sterk PJ, et al. Fluctuations and determinism of respiratory impedance in asthma and chronic obstructive pulmonary disease. J Appl Physiol 2010; 109: 1582-1591.

8 Que CL, Kenyon CM, Olivenstein R, et al. Homeokinesis and short-term variability of human airway caliber. J Appl Physiol 2001; 91: 1131-1141.

9 Frey U, Brodbeck T, Majumdar A, et al. Risk of severe asthma episodes predicted from fluctuation analysis of airway function. Nature 2005; 438: 667-670.

10 Pilgram B, Schappacher W, Löscher W, et al. Application of the correlation integral to respiratory data of infants during REM sleep. Biol Cybern 1995; 72: 543-551.

11 Frey U, Silverman M, Barabási AL, et al. Irregularities and power law distributions in the breathing pattern in preterm and term infants. J Appl Physiol 1998; 85: 789-797.

12 Small M, Judd K, Lowe M, et al. Is breathing in infants chaotic? Dimension estimates for respiratory patterns during quiet sleep. J Appl Physiol 1999; 86: 359-376.

13 Patzak A, Foitzik B, Mrowka R, et al. Time of measurement influences the variability of tidal breathing parameters in healthy and sick infants. Respir Physiol 2001; 128: 187-194.

14 Seppä V-P, Hyttinen J, Viik J. A method for suppressing cardiogenic oscillations in impedance pneumography. Physiol Meas 2011; 32: 337-345.

15 Seppä V-P, Hyttinen J, Uitto M, et al. Novel electrode configuration for highly linear impedance pneumography. Biomed Tech (Berl) 2013; 58: 35-38.

16 Seppä V-P, Pelkonen AS, Kotaniemi-Syriänen A, et al. Tidal breathing flow measurement in awake young children by using impedance pneumography. J Appl Physiol 2013; 115: 1725-1731.

17 Guilbert TW, Morgan WJ, Zeiger RS, et al. Atopic characteristics of children with recurrent wheezing at high risk for the development of childhood asthma. J Allergy Clin Immunol 2004; 114: 1282-1287.

18 Malmberg LP, Pelkonen A, Poussa T, et al. Determinants of respiratory system input impedance and bronchodilator response in healthy Finnish preschool children. Clin Physiol Funct Imaging 2002; 22: 64-71.

19 Malmberg LP, Mäkelä MJ, Mattila PS, et al. Exercise-induced changes in respiratory impedance in young wheezy children and nonatopic controls. Pediatr Pulmonol 2008; 43: 538-544.

20 Beydon N, Davis SD, Lombardi E, et al. An Official American Thoracic Society/European Respiratory Society statement: pulmonary function testing in preschool children. Am J Respir Crit Care Med 2007; 175: 1304-1345.

21 Shee CD, Ploy-Song-Sang Y, Milic-Emili J. Decay of inspiratory muscle pressure during expiration in conscious humans. J Appl Physiol 1985; 58: 1859-1865.

22 Morris MJ, Madgwick RG, Frew AJ, et al. Breathing muscle activity during expiration in patients with chronic airflow obstruction. Eur Respir J 1990; 3: 901-909.

23 Poon C-S, Barahona M. Titration of chaos with added noise. Proc Natl Acad Sci U S A 2001; 98: 7107-7112.

24 Meier $\mathrm{T}$, Luepschen $\mathrm{H}$, Karsten J, et al. Assessment of regional lung recruitment and derecruitment during a PEEP trial based on electrical impedance tomography. Intensive Care Med 2007; 34: 543-550.

25 Seppä V-P, Uitto M, Viik J. Tidal breathing flow-volume curves with impedance pneumography during expiratory loading. Conf Proc IEEE Eng Med Biol Soc 2013; 2013: 2437-2440.

26 van der Ent CK, van der Grinten CP, Meessen NE, et al. Time to peak tidal expiratory flow and the neuromuscular control of expiration. Eur Respir J 1998; 12: 646-652.

27 Brand PL, Duiverman EJ, Postma DS, et al. Peak flow variation in childhood asthma: relationship to symptoms, atopy, airways obstruction and hyperresponsiveness. Dutch CNSLD Study Group. Eur Respir J 1997; 10: 1242-1247.

28 Bridge PD, Lee H, Silverman M. A portable device based on the interrupter technique to measure bronchodilator response in schoolchildren. Eur Respir J 1996; 9: 1368-1373.

29 Merkus PJ, Mijnsbergen JY, Hop WC, et al. Interrupter resistance in preschool children: measurement characteristics and reference values. Am J Respir Crit Care Med 2001; 163: 1350-1355. 
30 Lall CA, Cheng N, Hernandez P, et al. Airway resistance variability and response to bronchodilator in children with asthma. Eur Respir J 2007; 30: 260-268.

31 Suki B. Fluctuations and power laws in pulmonary physiology. Am J Respir Crit Care Med 2002; 166: 133-137.

32 Quan SF, Goodwin JL, Babar SI, et al. Sleep architecture in normal Caucasian and Hispanic children aged 6-11 years recorded during unattended home polysomnography: experience from the Tucson Children's Assessment of Sleep Apnea Study (TuCASA). Sleep Med 2003; 4: 13-19.

33 Bellia V, Cuttitta G, Insalaco G, et al. Relationship of nocturnal bronchoconstriction to sleep stages. Am Rev Respir Dis 1989; 140: 363-367.

34 Terrill PI, Wilson SJ, Suresh S, et al. Attractor structure discriminates sleep states: recurrence plot analysis applied to infant breathing patterns. IEEE Trans Biomed Eng 2010; 57: 1108-1116.

35 Rostig S, Kantelhardt JW, Penzel T. Nonrandom variability of respiration during sleep in healthy humans. Sleep 2005; 28: 411-417.

36 Long X, Yang J, Weysen $\mathrm{T}$, et al. Measuring dissimilarity between respiratory effort signals based on uniform scaling for sleep staging. Physiol Meas 2014; 35: 2529.

37 Kales A, Kales JD, Sly RM, et al. Sleep patterns of asthmatic children: all-night electroencephalographic studies. J Allergy 1970; 46: 300-308.

38 Fitzpatrick MF, Engleman H, Whyte KF, et al. Morbidity in nocturnal asthma: sleep quality and daytime cognitive performance. Thorax 1991; 46: 569-573. 